\title{
A multicenter retrospective cohort study of ketogenic diet therapy in 481 children with infantile spasms
}

\author{
Yuanzhen $\mathrm{Ye}^{1 \dagger}$, Dan Sun ${ }^{2 \dagger}$, Hua $\mathrm{Li}^{3 \dagger}$, Jianmin Zhong ${ }^{4 \dagger}$, Rong Luo ${ }^{5 \dagger}$, Baomin $\mathrm{Li}^{6 \dagger}$, Dengna Zhu ${ }^{7}$, Dan Li ${ }^{8}$, \\ Shaoping Huang ${ }^{8}$, Yuwu Jiang ${ }^{9}$, Nong Xiao ${ }^{10}$, Yucai Chen ${ }^{11}$, Yuqin Zhang ${ }^{12}$, Mei Yu' ${ }^{1}$, Xiaoyun Shen ${ }^{1}$, Li Gao ${ }^{13}$, \\ Guo Zheng ${ }^{14}$, Congmin Zhao ${ }^{15}$, Baoqiang Yuan ${ }^{16^{*}}$, Jianxiang Liao ${ }^{1 *} \mathbb{O}$, Jiong Qin ${ }^{17^{*}}$ and CAAE KD group
}

\begin{abstract}
Background: Ketogenic diet (KD) therapy is one of the main treatments for drug-resistant epilepsy. However, the KD therapy has been applied in only a small number of infantile spasm cases. In this large multicenter study, we investigated the efficacy of KD therapy in the treatment of infantile spasms.

Methods: In this retrospective, multicenter cohort study, clinical data from main epilepsy centers were analyzed. Patients were classified into different groups according to age, type of drug and whether glucocorticoid was used before initiation of KD.

Results: From October 2014 to March 2020, 481 patients (308 males and 173 females) with infantile spasms were treated with the KD therapy. The age of the patients ranged from 2 months to 20 years, with a mean age of 1 year and 10 months. The number of anti-seizure medications (ASMs) used before KD initiation ranged 0-6, with a median of 3 . In different time from initiation(1, 3, 6, and 12 months), the rates of seizure freedom after $\mathrm{KD}$ were 6.9, 11.6, 16.0 and $16.8 \%$, respectively $\left(X^{2}=27.1772, P<0.0001\right)$. There was a significant difference in the rate of seizure freedom between 3 months and 1 month $\left(X^{2}=6.5498, P=0.0105\right)$ groups, and 6 months and 3 months $\left(X^{2}=3.8478, P=0.0498\right)$ groups, but not between 12 months and 6 months $\left(X^{2}=0.1212, P=0.7278\right)$ groups. The rates of effectiveness were $44.7,62.8,49.1$ and $32.0 \%\left(X^{2}=93.2674, P<0.0001\right)$, respectively. The retention rates were $94.0,82.5,55.7$ and $33.1 \%$ $\left(X^{2}=483.7551, P<0.0001\right)$, correspondingly. The rate of effectiveness and the retention rate of KD were significantly different among the 1, 3, 6 and 12 months. KD treatment was the first choice in 25 patients (5.2\%), 55 patients (11.4\%) started KD after the failure of the first ASM, 158 patients (32.8\%) started KD after the failure of the second ASM, 157 patients (32.6\%) started KD after the failure of the third drug, and 86 patients (17.9\%) started KD after the failure of the fourth and more. The KD effect was not related to the number of ASMs used before KD startup $(P>0.05)$. Two hundred and eighteen patients (45.3\%) failed to respond to corticotropin or glucocorticoid before initiation. There
\end{abstract}

\footnotetext{
*Correspondence: Yuanbqiang@Hotmail.com; liaojianxiang@vip.sina.com; qinjiong@pkuph.edu.cn

†Yuanzhen Ye, Dan Sun, Hua Li, Jianmin Zhong, Rong Luo and Baomin Li

contributed equally to this work.

1 Shenzhen Children's Hospital, Shenzhen 518038, China

${ }^{16}$ The Affiliated Hospital of Xuzhou Medical University, Xuzhou 221004,

China

${ }^{17}$ Peking University People's Hospital, Beijing 100044, China

Full list of author information is available at the end of the article
} give appropriate credit to the original author(s) and the source, provide a link to the Creative Commons licence, and indicate if changes were made. The images or other third party material in this article are included in the article's Creative Commons licence, unless indicated otherwise in a credit line to the material. If material is not included in the article's Creative Commons licence and your intended use is not permitted by statutory regulation or exceeds the permitted use, you will need to obtain permission directly from the copyright holder. To view a copy of this licence, visit http://creativecommons.org/licenses/by/4.0/. 
was no significant difference in the effectiveness rate at different time points between the group of KD therapy after glucocorticoid failure and the group after non-hormone failure $\left(X^{2}=0.8613, P=0.8348\right)$. The rate of adverse events of $K D$ in $1,3,6$, and 12 months after $K D$ initiation were 22.3, 21.7, 16.8 and 6.9\%, respectively. The adverse events mainly occurred during the first 3 months of KD, and the main adverse events were gastrointestinal disturbance and constipation.

Conclusions: The efficacy of the KD treatment for infantile spasms was not affected by age, medication, and glucocorticoid use before initiation. KD is one of the effective treatments for infantile spasms.

Trial registration: ChiCTR-IIR-16008342. Registered on 22 April, 2016 - Retrospectively registered, https://www.chictr. org.cn.

Keywords: Epilepsy, Infantile spasms, Ketogenic diet, Adverse events, Effectiveness rate, West syndrome, Multicenter study, Retrospective, Cohort

\section{Background}

Infantile spasms (West syndrome) is a clinically common epilepsy syndrome of multiple causes, with typical manifestations of spasms, hypsarrhthmia (typical finding on interictal electroencephalogram [EEG]), and psychomotor development delay [1]. Although corticotropin $(\mathrm{ACTH})$ and vigabatrin are used as the first-line treatment for infantile spasms, the epileptic spasms of some patients could not be effectively controlled. In addition, although $\mathrm{ACTH}$ is recommended as the first-line treatment in many countries, it has a high incidence of side effects and infantile spasms may relapse in nearly $33-50 \%$ of patients, which limits its generalized use [2-4]. Ketogenic diet (KD), a dietary model with high fat, low carbohydrate, and appropriate protein and other nutrients, can not only control seizures, but also improve EEG, development and cognition, thus having become an effective non-drug treatment for drug-resistant infantile spasms [5-7]. KD has developed rapidly since its introduction in China in 2004 [8], and KD therapy has been gradually carried out throughout the country. In this study, we set out to evaluate the effect and safety of KD therapy in a large sample size of infantile spasms in 17 tertiary hospitals in China.

\section{Materials and methods Study design and patients}

This study was a prospectively registered clinical trial carried out in a multicenter cohort. Data were collected by trained pediatric epileptologists, including 481 patients with infantile spasms treated with KD therapy. Nutritionists from multiple centers receive uniform training. A uniform case report form was used in these centers. This study was approved by the Medical Ethics Committee of Shenzhen Children's Hospital, with a Clinical trial registration No. of ChiCTR-IIR-16008342.

\section{Participant inclusion/exclusion criteria}

The inclusion criteria for participants were: (1)Meeting the diagnostic criteria of infantile spasms, having clinical epileptic spasms, displaying hypsarrhythmia on EEG and psychomotor retardation; (2)having not received KD therapy before. The exclusion criteria included: (1)Patients with severe malnutrition; digestive, cardiovascular, respiratory, liver, or urinary system disease; or congenital immunodeficiency; or genetic metabolic diseases as KD contraindications. The relative contraindications to KD therapy included propofol concurrent use (a higher risk for propofol infusion syndrome); poor cooperation of patient's parent or caregiver; identification of a surgical epileptogenic zone by semiology, video-EEG monitoring and neuroimaging; inability to maintain adequate nutrition. The absolute KD therapy contraindications included porphyria; carnitine palmitoyltransferase (CPT) I or II deficiency; pyruvate carboxylase deficiency; carnitine translocase deficiency; carnitine deficiency (primary); and beta-oxidation defects including long-chain acyl dehydrogenase deficiency, long-chain 3-hydroxyacyl-CoA deficiency, medium-chain 3-hydroxyacyl-CoA deficiency, medium-chain acyl dehydrogenase deficiency, and short-chain acyl dehydrogenase deficiency. (2)Active vomiting, fever or infectious diseases; (3) transplantation, stem cell therapy, or transfusion of allogeneic blood products within half a year prior to KD initiation, or hemolytic diseases, etc.

\section{Data collection and statistics}

Patient data were collected from 2014 to 2020. Case report forms included a basic information form, clinical medical records, a KD initiation observation form, a KD follow-up form and a form of examination results.

\section{KD therapy}

Before diet initiation, detailed health education was provided to the patients's guardians and written informed consent was given by the guardians. According to the clinical standardized guidelines of $K D$, the KD therapy was initiated with a ratio of $2: 1$ in the inpatient or outpatient department without fasting, 
and the calorie and protein requirements were calculated according to the recommended level for patients at a specific age. One third of the recommended total calories were provided on the first day of the therapy, and two thirds were provided on the second day, and full recommended total calories were provided on the third day. Ketogenic operation was simplified by using the ketogenic series products from Shenzhen Zeneca Biotechnology Co., LTD., to assist in the implementation of KD. Transition speed was adjusted, combined with some home-made meals according to the patient's condition to make food palatable. During KD treatment, daily multivitamins, minerals, vitamin D and calcium were supplemented, and potassium citrate was used to prevent kidney stones. Vital signs, urine ketone, blood ketone and blood glucose were monitored, and seizures, diet and adverse events were recorded every day. At 2-4 weeks after initiation, the proportion of $\mathrm{KD}$ was adjusted according to the level of ketone body, the frequency of seizures and the dietary tolerability, etc. For patients with frequent seizures and good tolerance, the proportion was recommended to increase to $4: 1$. Within 1 to 3 months of KD treatment, the original anti-seizure medication (ASM) regimen remained unchanged, and then the drugs were adjusted according to the seizure status and the doctor's advice, and only one medication was adjusted at a time. Clinical dietitians performed fine regulation and were responsible for long-term management of patients. The patients were followed up daily during the initiation of $K D$, weekly within the first month of $K D$, and every 2 to 4 weeks thereafter. In the first, second, third, and every 3 months afterward, the patients were followed up in the hospital to review their height, weight, blood biochemistry, urinary system ultrasound, bone development and EEG. Patients were followed for 1 year or more.

\section{Outcome}

The outcome measures were seizure frequency and adverse events during KD. Seizures recorded by guardians during 4 weeks prior to KD treatment were used as the baseline, and the effect and retention at 1, 3, 6, and 12 months after the initiation of KD were recorded.

The effect was determined as seizure-free $(100 \%$ reduction in seizure frequency, for at least 1 month), effective (equal or more than $50 \%$ reduction in seizure frequency), and ineffective (less than $50 \%$ reduction in seizure frequency). The rate of seizure freedom refers to the percentage of seizure-free patients in total patients enrolled; the effectiveness rate refers to the percentage of patients with an equal or more than $50 \%$ seizure reduction in total patients enrolled; the ineffectiveness rate refers to the percentage of patients with a $<50 \%$ seizure reduction in total patients enrolled.

\section{Data analysis}

Data analysis was performed using the SPSS 23.0 software. The rank sum test (Kruskal-Wallis $H$ ) was used to evaluate the difference of retention rate, anti-seizure effects and adverse events of KD therapy among different hospitals. The Chi-square test was used to analyze data on the reduction of seizures, the effectiveness rate, and retention rate reported at different time points among the groups.

\section{Results}

\section{Demographic features}

A total of 481 patients (308 males and 173 females) were included in the study, with a KD initiation age of 2 months to 20 years old (median age, 1 year and 3 months). The baseline frequency of epilepsy was 0.1 to 130 times/day, with a median of 6 times/day. The number of ASMs at KD initiation was $0-6$, with a median of 3 . The duration of continuous KD ranged 1 month to 5 years, with a median duration of 6 months. At the last follow-up, KD was still maintained in 31 patients, the longest duration was 4 years and 4 months, and the median duration was 2 years.

\section{Effect and retention rate}

The improvement of seizures in patients with infantile spasms treated with KD is shown in Table 1. At 1, 3, 6 and 12 months after KD initiation, the rates of seizure freedom were $6.9 \%, 11.6 \%, 16.0 \%$ and $16.8 \%$, respectively $\left(\chi^{2}=27.1772, P<0.0001\right)$, with a significant difference between 3 months and 1 month $\left(x^{2}=6.5498\right.$, $P=0.0105)$, and between 6 months and 3 months $\left(x^{2}=3.8478, P=0.0498\right)$, but not between 12 months and 6 months $\left(\chi^{2}=0.1212, P=0.7278\right)$. The results suggest that it is necessary to adhere to the KD therapy for 3-6months, and that if seizure reduction was less than $50 \%$ at 6 months, then the seizure reduction was largely unlikely to significantly increase at 12 months.

Table 1 Effectiveness and retention rate of KD in 481 patients with infantile spasms at 1, 3, 6 and 12 months of follow-up

\begin{tabular}{lllll}
\hline Effect/time points & 1 month & 3 months & $\mathbf{6}$ months & 12 months \\
\hline Seizure-free $n(\%)$ & $33(6.9)$ & $56(11.6)$ & $77(16.0)$ & $81(16.8)$ \\
$\begin{array}{l}\text { Effective(seizure } \\
\text { reduction } \geq 50 \%)\end{array}$ & $215(44.7)$ & $302(62.8)$ & $236(49.1)$ & $154(32.0)$ \\
$\begin{array}{l}n(\%) \\
\text { Retention rate } n(\%)\end{array}$ & $452(94.0)$ & $397(82.5)$ & $268(55.7)$ & $159(33.1)$ \\
\hline
\end{tabular}


The rates of effectiveness at 1, 3, 6 and 12 months after KD initiation were $44.7 \%, 62.8 \%, 49.1 \%$ and $32.0 \%$, respectively $\left(\chi^{2}=93.2674, P<0.0001\right)$, and the retention rates were $94.0 \%, 82.5 \%, 55.7 \%$ and $33.1 \%$, respectively $\left(\chi^{2}=483.7551, P<0.0001\right)$. Both the rate of effectiveness and the retention rate were significantly different among the time points of $1,3,6$ and 12 months. The rate of effectiveness increased at 3 months compared to 1 month, and then decreased gradually at 6 and 12 months. The retention rate decreased at 3, 6 and 12 months. There was no significant difference in the rate of effectiveness of KD therapy among different hospitals at 3 months of $\mathrm{KD}(\mathrm{H}=19.085, P>0.05)$.

\section{Age and effect}

A majority $(87.8 \%)$ of the patients were aged 03 years. There was no significant difference in the retention rate at 3, 6 and 12 months of KD (KD3, KD6, KD12) among different age groups [age group included:0-1,1-3,3-6,6-1 0,10 -20years] (KD3, $\chi^{2}=1.452$; KD6, $\chi^{2}=1.123$; KD12, $X^{2}=0.803$; all $\left.P>0.05\right)$. There was also no significant difference in the rate of effectiveness at KD1, KD3, KD6 and KD12 among different age groups [age group inclu ded:0-1,1-3,3-6,6-10,10-20 years](KD1, $\chi^{2}=1.549 ;$ KD3, $\chi^{2}=1.576$; KD6, $\chi^{2}=3.173$; KD12, $\chi^{2}=0.771$; all $\left.P>0.05\right)$.

\section{Concurrent medications}

In the newly diagnosed infantile spasms, KD treatment was preferred in 25 patients (5.2\%), and 55 patients (11.4\%) started KD after the failure of the first ASM, 158 patients (32.8\%) started KD after the failure of the second

Table 2 The efficiency of KD in patients with infantile spasms with prior use of different ASMs

\begin{tabular}{llllll}
\hline & No ASMs & $\mathbf{1}$ drug & 2 drugs & 3 drugs & $\geq$ 4 drugs \\
\hline$n(\%)$ & $25(5.2)$ & $55(11.4)$ & $158(32.8)$ & $157(32.6)$ & $86(17.9)$ \\
1 month(a) & $14(56.0)$ & $26(47.3)$ & $68(43.0)$ & $65(41.4)$ & $33(38.4)$ \\
3 months(a) & $12(48.0)$ & $23(41.8)$ & $85(53.8)$ & $89(56.7)$ & $41(47.7)$ \\
6 months(a) & $10(40.0)$ & $19(34.5)$ & $65(41.1)$ & $65(41.4)$ & $27(31.4)$ \\
12 months(a) & $7(28.0)$ & $12(21.8)$ & $46(29.1)$ & $43(27.4)$ & $14(16.3)$
\end{tabular}

Note:ASMs anti-seizure medications; a: The response rate in the population with maintenance or reduced ASMs and more than $50 \%$ seizure reduction. Data are provided as $n(\%)$
ASM, 157 patients (32.6\%) started KD after the failure of the third ASM, and 86 patients (17.9\%) started KD after the failure of the fourthASM and more (Table 2). The number of patients with decreased ASMs was higher than those with increased ASMs (data unshown, $P<0.05)$. At the same KD time point, the KD effect was not related to the number of ASMs used before KD initiation $(P>0.05)$. In 218 patients $(45.3 \%)$ who failed to respond to $\mathrm{ACTH}$ or glucocorticoid treatment (referred to as hormone failure hereinafter) before KD initiation, the rates of effectiveness of KD at 1, 3, 6 and 12 months were $48.2 \%, 64.2 \%, 50.0 \%$ and $35.3 \%$, respectively (Table 3). These rates were close to those in the whole sample, and similarly, the rate of effectiveness increased at 3 months compared to 1 month, and then decreased gradually $\left(\chi^{2}=36.6057, P<0.0001\right)$. There was no significant difference in the effectiveness rate of KD therapy at different time points after hormone failure, compared with the overall effectiveness rate and the effectiveness rate of patients without hormone treatment $\left(\chi^{2}=0.3047\right.$, $\left.P=0.9591 ; \chi^{2}=0.8613, P=0.8348\right)$.

\section{Adverse events and withdrawal}

Adverse events during KD mainly occurred in the first 3 months. The common adverse events were gastrointestinal disturbances (nausea, vomiting, abdominal pain, diarrhea, etc.) and constipation. Other adverse events included fever, cold, fatigue or mental retardation, cough, anorexia, pneumonia, symptomatic hypoglycemia, enteritis, allergy, high triglyceride or cholesterol, kidney stones, low bone mineral density and hypoproteinemia (Table 4). The overall incidence was different among different time points $\left(\chi^{2}=15.382, P<0.05\right)$. The incidence of gastrointestinal disturbances was significantly different among different time points $\left(\chi^{2}=16.613, P<0.05\right)$. The incidence of gastrointestinal disturbances was $9.1 \%$ in the first month of KD, and significantly decreased at 6 and 12 months $\left(X^{2}=8.706, P<0.05 ; X^{2}=9.888, P<0.05\right)$. The incidence of anorexia increased at 3 months compared to 1 month $\left(X^{2}=10.585 ; \quad P<0.05\right)$. Hypoproteinemia mainly occurred at 3 months of $K D$, and there was significant difference among different time points $\left(\chi^{2}=8.770\right.$; $P<0.05)$. There was no significant difference in the incidence of other adverse events among different time points $(P>0.05)$.

Table 3 Effect of KD in 218 patients with infantile spasms after ACTH or glucocoticoid failure

\begin{tabular}{|c|c|c|c|c|c|c|c|}
\hline \multicolumn{2}{|l|}{1 month } & \multicolumn{2}{|l|}{3 months } & \multicolumn{2}{|l|}{6 months } & \multicolumn{2}{|l|}{12 months } \\
\hline Retention & Effectiveness & Retention & Effectiveness & Retention & Effectiveness & Retention & Effectiveness \\
\hline 210 (96.3) & $105(48.2)$ & $186(85.3)$ & $140(64.2)$ & $122(56.0)$ & $109(50.0)$ & $78(35.8)$ & 77 (35.3) \\
\hline
\end{tabular}

Data are provided as $n(\%)$ 
Table 4 Adverse events in patients with infantile spasms at different time points of follow-up

\begin{tabular}{|c|c|c|c|c|}
\hline Adverse events, $n(\%)$ & 1 month & 3 months & 6 months & 12 months \\
\hline Gastrointestinal disdurbance* & $41(9.1)$ & $24(6.0)$ & $8(3.0)$ & $2(1.3)$ \\
\hline Constipation & $22(4.9)$ & $13(3.3)$ & $7(2.6)$ & $3(1.9)$ \\
\hline Fever & $10(2.2)$ & $9(2.3)$ & $1(0.4)$ & $1(0.6)$ \\
\hline Cold & $6(0.66)$ & $2(0.5)$ & $3(1.1)$ & 0 \\
\hline Lack of energy or mental health & $5(1.1)$ & 0 & 0 & 0 \\
\hline Cough & $3(0.7)$ & $4(1.0)$ & 0 & 0 \\
\hline Anorexia * & $4(0.9)$ & $10(2.5)$ & $13(4.9)$ & $4(2.5)$ \\
\hline Pneumonia & $4(0.9)$ & $9(2.3)$ & $6(2.2)$ & 0 \\
\hline Symptomatic hypoglycemia & $1(0.2)$ & $3(0.8)$ & $1(0.4)$ & 0 \\
\hline Enteritis & $1(0.2)$ & 0 & 0 & 0 \\
\hline Allergy & $1(0.2)$ & $1(0.3)$ & 0 & 0 \\
\hline $\begin{array}{l}\text { High triglycerides } \\
\text { or cholesterol }\end{array}$ & $2(0.4)$ & $4(1.0)$ & $2(0.8)$ & 0 \\
\hline Kidney stones & 0 & 0 & 0 & $1(0.6)$ \\
\hline Low bone mineral density & $1(0.2)$ & $1(0.3)$ & $2(0.8)$ & 0 \\
\hline Hypoproteinemia* & 0 & $6(1.5)$ & $2(0.8)$ & 0 \\
\hline Continuation of KD (n) & 452 & 397 & 268 & 159 \\
\hline Total (\%) & $101 / 452(22.3)$ & $86 / 397(21.7)$ & $45 / 268(16.8)$ & $11 / 159(6.9)$ \\
\hline
\end{tabular}

Table 5 Causes of KD withdrawal in 481 infantile spasm patients within 12 months

\begin{tabular}{llll}
\hline Reasons of withdrawal & $\boldsymbol{n}(\%)$ & Reasons of withdrawal & $\boldsymbol{n}$ (\%) \\
\hline Ineffectiveness & $96(20.0)$ & Death & $8(1.7)$ \\
Loss of follow-up & $47(9.8)$ & Fever & $7(1.5)$ \\
Poor compliance & $35(7.3)$ & Recurrence of epilepsy & $7(1.5)$ \\
Adding drug therapy & $14(2.9)$ & Surgery & $7(1.5)$ \\
Change hospitals for treat- & $6(1.2)$ & Bronchitis & $2(0.4)$ \\
ment & & & $2(0.4)$ \\
Effective attack control & $11(2.3)$ & Cold & $1(0.2)$ \\
Anorexia & $22(4.6)$ & Allergy & $1(0.2)$ \\
Gastrointestinal disdur- & $21(4.4)$ & Constipation & $1(0.2)$ \\
bance & & & $9(1.8)$ \\
Pneumonia & $16(3.3)$ & Kidney stones \\
Hypoimmunity & $9(1.9)$ & Others & \\
Total (\%) & $322(66.9)$ & &
\end{tabular}

All patients on the KD therapy were followed up. Among them, 159 patients maintained KD for more than 1 year, and 322 patients maintained KD for less than 1 year. Among the 159 patients, 117 had maintained KD for 2 years, 34 had maintained KD for 3 years, and seven had maintained KD for 4 years or more.

The reasons for KD withdrawal within 1 year included poor effect (96 patients, 20.0\%), lost to follow-up (47 patients, 9.8\%), poor compliance or parents' failure to cooperate (35 patients, $7.3 \%$ ), and scheduled treatment plan for effective control of seizures (11 patients, $2.3 \%$ ).
The main adverse event that caused KD withdrawal was gastrointestinal disturbances in 21 patients (4.4\%) (Table 5).

The causes of death of eight patients were heart failure (one patient), status epilepticus (two patients), increased seizure and the family gave up rescue (one patient), high fever and infection (two patients), virus infection (one patient), and sudden illness and death on the way to the hospital after 1 month of seizure control, without definite diagnosis (one patient). There was no direct causal relationship with KD therapy.

\section{Discussion}

In this multicenter study, we evaluated KD in the treatment of infantile spasms. KD treatment was the first choice for new diagnosis patients, accounting for $5.2 \%$. The proportion of patients who started KD after treatment failure of the first, second, third, and fourth ASMs or more were $11.4 \%, 32.8 \%, 32.6 \%$ and $17.9 \%$, respectively. The rates of seizure freedom at 1, 3, 6 and 12 months were $6.9 \%, 11.6 \%, 16.0 \%$ and $16.8 \%$, respectively, with no significant difference between 6 months and 12 months. The rates of effectiveness were $44.7 \%, 62.8 \%, 49.1 \%$ and $32.0 \%$, respectively. The retention rates at these time points were $94.0 \%, 82.5 \%, 55.7 \%$ and $33.1 \%$, correspondingly. There was no correlation between the effectiveness rate and the number of ASMs used before ketogenic treatment, whether being hormone failure or not.

The rate of seizure freedom of KD treatment for 1 month was $6.9 \%$, which was lower than that reported 
by Pires et al. (35.0\%) [9] and reported by Cao Dezhi et al. (85.7\%) [10]. However, our results also showed that nearly half of the patients with infantile spasms benefited from KD within 1 month. Compared with patients with intractable epilepsy, the effect of KD on infantile spasms occurred within 2-4 weeks after initiation $[6,10]$. Therefore, the seizure freedom rate at 1 month might be one of the indicators to evaluate whether KD is carried out reasonably in infantile spasms. Our data showed that the seizure freedom rate was increased at 3 and 6 months, with no significant difference between 12 and 6 months, suggesting that it is meaningful to maintain KD therapy for at least 3 months, and for patients who did not show $\mathrm{KD}$ efficacy, it is essential to extend the duration up to 6 months. For patients who still did not show response to KD at 6 months, KD therapy could be stopped. Randomized controlled trials have shown that $11 / 46$ patients had seizure freedom effect at 4 weeks with KD therapy [11], and the low diet ratio initiated in this study might be related to the low rate of seizure freedom at 1 month. Increasing the diet proportion to $3: 1$ or $4: 1$, such as the classic $\mathrm{KD}$, might improve the seizure freedom rate and the effectiveness rate [12].

The effectiveness rate of KD in the treatment of infantile spasms at 3 months of KD was $62.8 \%$, which was similar to previous reports $[5,6,13,14]$. The effectiveness rate was higher than that in children with epilepsy at 3 months of KD reported in a prospective study in 2013 (37.1\%) [15], but our seizure freedom rate $(11.6 \%)$ was lower than the reported $21.7 \%$. The study published by Li et al. [16] in 2013 showed that the effectiveness rate in 16 patients with infantile spasms after 3 months of KD treatment was 81\% (13/16); Zhou et al. [17] reported a seizure freedom rate of $37.5 \%$ in children with late-onset epileptic spasms after 3 months of KD. The reasons for the low rate of seizure freedom in this study may be related to the KD protocol, patient compliance, infantile spasms severity, etc. At 6 and 12 months of KD, the effectiveness rates of KD in the treatment of infantile spasms were $49.1 \%$ and $32.0 \%$, respectively. In addition, the effective treatment of KD may improve the compliance of patients. Most of the patients who maintained KD till 12 months showed KD effectiveness. There was no significant difference in the effectiveness rate among the 17 hospitals included in this study $(P>0.05)$. The results represent the current situation of KD development, suggesting high levels of standardization and uniformity of KD treatment in various institutions.

In this study, $83.3 \%$ of patients started KD after use of two or more ASMs, suggesting that KD is still used mainly in the treatment of refractory epilepsy in the participating hospitals. In addition, $11.4 \%$ of children used KD after failure of one kind of ASM treatment, and 5.2\% used KD directly after new diagnosis. Although there was no significant difference in the effectiveness rate of patients with different numbers of ASMs $(P>0.05)$, KD improved cognition and behavior in these patients. Early KD may benefit brain function. After the failure of $\mathrm{ACTH}$ or glucocorticoid treatment, $45.3 \%$ of patients switched to $\mathrm{KD}$ treatment, and the effectiveness rate of $\mathrm{KD}$ for 3 months was $62.4 \%$. This rate was similar to the 4 -month effectiveness rate $(54.1 \%)$ of $\mathrm{KD}$ in refractory infantile spasms, which was initiated by 10 centers after failure of ACTH or glucocorticoid treatment, as reported by Zhang et al. [18]. Therefore, we believe that KD may improve the condition after the failure of ACTH or glucocorticoid treatment, and Zhang et al. [18] also proposed that the effect of KD treatment is better than that of further oral ASMs. Meanwhile, it has been reported that the efficacy of $\mathrm{KD}$ in patients with newly diagnosed infantile spasms was not worse than that of ACTH, and the side effects were less. Therefore, some authors suggested KD as the first-line treatment for infantile spasms $[9,18,19]$, indicating the value of early use of KD in patients with infantile spasms, or even taking KD as the first-line treatment.

This study found that the effectiveness rate and the retention rate of KD were similar in different age groups, which was consistent with previous studies [14, 20, 21]. The 2018 international guidelines recommend that infants under 2 years are the ideal age group for KD [7]. Among our infantile spasms patients, 35.6\% were infants under 1 year and the minimum age was 2 months, suggesting that $\mathrm{KD}$ is safe in children over 2 months. The young children in this study were from 17 centers respectively, which revealed that each epilepsy center had accumulated experience in developing KD for infants and children of all ages. The retention rate is an important measure in evaluating the success of $\mathrm{KD}$. In this study, the retention rates of $\mathrm{KD}$ at $1,3,6$ and 12 months after initiation were $94.0 \%, 82.5 \%, 55.7 \%$ and $33.1 \%$, respectively. The retention rates of each period were similar to or slightly higher than those reported in relevant literature [14, 16, 22-24]. Based on this report involving 17 epilepsy centers, although there was no overall difference in the efficiency and retention rate among centers $(P>0.05)$, in reality, the concept of KD management, the degree of family cooperation and the freedom of patients' choice of medical treatment can all affect the retention rate. The retention rate further decreased from 3 months of KD, which may be related to the fact that the effect of KD was usually evaluated at 3-6 months. It is often more difficult to adhere to KD when the patient's family believe that it is ineffective or the effect does not meet the expectation. In the withdrawal analysis, $20.0 \%$ of withdrawal were due to poor seizure improvement, which was similar to the previously reported results [6]; $17.1 \%$ of patients stopped KD 
due to family loss of follow-up and poor compliance / poor parental cooperation, which may be related to the difficulty in understanding KD by the elderly of some families or the difficulty of young parents in KD implementation. At the same time, $4.1 \%$ of patients received add-on of new ASMs or hospital transferring treatment, which not only reflected the flexibility of medical care, but also indicated that the effect of short-term KD might not meet the expectations of their families. Therefore, how to improve the effectiveness of KD individually, do a good job in the whole process of management, make evaluation of KD quantifiable, and simplify the operation are key issues that should receive more attention and be solved at present, because the KD retention rate is an important prognostic factor for maintenance of the curative effect [6].

In our study, adverse events occurred in some patients, but most of them were transient and tolerable, which were improved by conservative treatment or dietary adjustment. The incidence of hypoproteinemia, kidney stones, high triglycerides or cholesterol was low, and throughout the KD period, the incidence of adverse events was also low $(<2.0 \%)$. Good diet management and a reasonable scheme can reduce the occurrence of adverse events and increase the safety. Seizure control effect, expectation and dietary compliance play a key role in the continuation of KD. During KD, 8 patients died from different causes. Although the deaths were not directly caused by $\mathrm{KD}$, careful observation is still needed during diet therapy to reduce complications and ensure a timely and appropriate treatment at its occurrence.

As a multicenter retrospective multicenter cohort study, the data might not be completely accurate. The long-term retention rate and the effectiveness rate, although similar to those reported in a prospective controlled study, were low in this study. In the future, multi-center large-sample clinical trials should be designed to study the related factors of retention rate and effectiveness, and further improve them.

\section{Conclusions}

In this study, we analyzed the effect and safety of KD treatment in 481 patients with infantile spasms from multicenter registered trial between 2014 to 2020. Most of the patients had drug-resistant epilepsy. At 1, 3, 6 and 12 months of $\mathrm{KD}$, the seizure freedom rates were $6.9 \%$, $11.6 \%, 16.0 \%$ and $16.8 \%$ respectively, and the retention rates were $94.0 \%, 82.5 \%, 55.7 \%$ and $33.1 \%$, correspondingly. In summary, KD is effective in the treatment of infantile spasms with mild and acceptable side effects, and thus is suitable for clinical application. The anti-seizure effect and cognition improvement of KD therapy need to be further studied.
Abbreviations

KD: Ketogenic diet; ASMs: Anti-seizure medications; ACTH: Corticotropin; EEG: Electroencephalogram.

\section{Acknowledgements}

We thank Professor Shichuo Li and Mr. Lirong Duan, Ms. Hui Zhang, China Association against Epilepsy for supporting and guiding the study. We appreciate and thank parents and children who consented for the ketogenic diet therapy.

\section{Authors' contributions \\ $J X L, J Q, B Q Y$ and $Y Z Y$ designed the study, and revised the manuscript. Other authors conducted the study. MY and JXL drafted the manuscript. The author(s) read and approved the final manuscript. \\ Funding \\ This study was supported by the Sanming Project of Medicine in Shenzhen (SZSM201812005); Shenzhen Key Medical Discipline Construction Fund (No. SZXK033), and Shenzhen Fund for Guangdong Provincial High Level Clinical Key Specialties (No.SZGSP012).}

Availability of data and materials

Data available upon request to JX L.

\section{Declarations}

\section{Ethics approval and consent to participate}

This study was approved by the Medical Ethics Committee of Shenzhen Children's Hospital, with a Clinical trial registration No. of ChiCTR-IIR-16008342. Informed consent was obtained from all patients in this study.

\section{Consent for publication}

Not applicable.

\section{Competing interests}

Author JQ is the editorial board member for Acta Epileptologica. Author $J Q$ was not involved in the journal's review of, or decisions related to this manuscript. JX L is a consultant of Shenzhen Zeneca Biotechnology Co., LTD. Shenzhen, China.

\section{Author details}

'Shenzhen Children's Hospital, Shenzhen 518038, China. ${ }^{2}$ Wuhan Children's Hospital, Wuhan 430019, China. ${ }^{3}$ Guangdong 999 Brain Hospital, Guangzhou 510515, China. ${ }^{4}$ Jiangxi Provincial Children's Hospital, Nanchang 330006, China. ${ }^{5}$ West China Second University Hospital, Sichuan University,

Chengdu 610044, China. ' Qilu Hospital of Shandong University, Jinan 250063,

China. ${ }^{7}$ Third Affiliated Hospital, Zhengzhou University, Zhengzhou 450015 ,

China. ${ }^{8}$ The Second Affiliated Hospital of Xi'an Jiaotong University,

Xian 710004, China. ${ }^{9}$ Peking University First Hospital, Beijing 100034, China.

${ }^{10}$ Children's Hospital of Chongqing Medical University, Chongqing 400015, China. ${ }^{11}$ Children's Hospital of Shanghai, Shanghai 20006, China. ${ }^{12}$ Tianjin Children's Hospital, Tianjing 300134, China. ${ }^{13}$ Henan Provincial People's Hospital, Zhengzhou 451161, China. ${ }^{14}$ Nanjing Children's Hospital, Nanjing 210008,

China. ${ }^{15}$ Xinqiao Hospital, Army Medical University, Chongqing 400037, China.

${ }^{16}$ The Affiliated Hospital of Xuzhou Medical University, Xuzhou 221004, China.

${ }^{17}$ Peking University People's Hospital, Beijing 100044, China.

Received: 7 September 2021 Accepted: 19 December 2021

Published online: 15 February 2022

\section{References}

1. Hrachovy RA, Frost JD. Infantile epileptic encephalopathy with hypsarrhythmia (infantile spasms/west syndrome). J Clin Neurophysiol. 2003;20(6):408-25. https://doi.org/10.1097/00004691-200311000-00004.

2. Kossoff EH, Hedderick EF, Turner Z, Freeman JM. A case-control evaluation of the ketogenic diet versus ACTH for new-onset infantile spasms. Epilepsia. 2010;49(9):1504-9. https://doi.org/10.1111/j.1528-1167.2008.01606.x. 
3. Lin HC, Young C, Wang PJ, Lee WT, Shen YZ. ACTH therapy for Taiwanese children with west syndrome -- efficacy and impact on long-term prognosis. Brain Dev. 2006;28(3):196-201. https://doi.org/10.1016/j.braindev. 2005.07.002.

4. Ito M. Extremely low-dose ACTH therapy for west syndrome in Japan. Brain Dev. 2001;23(7):635-41. https://doi.org/10.1016/s0387-7604(01) 00302-3.

5. Kossoff EH, Pyzik PL, Mcgrogan JR, Vining EPG, Freeman JM. Efficacy of the ketogenic diet for infantile spasms. Pediatrics. 2002;109(5):780-3. https://doi.org/10.1542/peds.109.5.780.

6. Eun $\mathrm{SH}$, Kang HC, Dong WK, Kim HD. Ketogenic diet for treatment of infantile spasms. Brain Dev. 2006;28(9):566-71. https://doi.org/10.1016/j. braindev.2006.03.011.

7. Kossoff EH, Zupec-Kania BA, Auvin S, Ballaban-Gil KR, Bergqvist AGC, Blackford R, et al. Optimal clinical management of children receiving dietary therapies for epilepsy: Updated recommendations of the International Ketogenic Diet Study Group. Epilepsia Open. 2018;3(2). https://doi. org/10.1002/epi4.12225.

8. Dezhi C, Xinguo L, Jianxiang L, Hu Y, Li B, Huang TS, et al. The ketogenic diet in the treatment of epileptic epilepsy. J Practical Pediatr. 2007;22(4):288-9.

9. Pires ME, llea A, Bourel E, Bellavoine V, Merdriu D, Berquin P, et al. Ketogenic diet for infantile spasms refractory to first-line treatments: an open prospective study. Epilepsy Res. 2013;105(1-2):189-94. https://doi. org/10.1016/j.eplepsyres.2012.11.009.

10. Cao D, Yan HU, Zhu Y, Zhao X, Li B, Chen L, et al. A randomized controlled study of corticotropin and ketogenic diet in the treatment of neonatal spasm. Chin J Clin Pediatr. 2011;18:1442-5.

11. Sharma S, Goel S, Kapoor D, Garg D, Panda I, Elwadhi A, et al. Evaluation of the Modified Atkins Diet for the Treatment of Epileptic Spasms Refractory to Hormonal Therapy: A Randomized Controlled Trial. J Child Neurol. 2021;36(8):686-91. https://doi.org/10.1177/08830738211004747.

12. Kim JA, Yoon JR, Lee EJ, Lee JS, Kim JT, Kim HD, et al. Efficacy of the classic ketogenic and the modified Atkins diets in refractory childhood epilepsy. Epilepsia. 2016;57(1):51-8. https://doi.org/10.1111/epi.13256.

13. Hong AM, Turner Z, Hamdy RF, Kossoff EH. Infantile spasms treated with the ketogenic diet:prospective single center experience in 104 consecutive infants. Epilepsia. 2010;51(8):1403-7. https://doi.org/10.1111/j.15281167.2010.02586.x.

14. Kayyali HR, Gustafson M, Myers T, Thompson L, Williams M, Abdelmoity A. Ketogenic diet efficacy in the treatment of intractable epileptic spasms. Pediatr Neurol. 2014;50(3):224-7. https://doi.org/10.1016/j.pediatrneurol. 2013.11.021.

15. Lin SF, Qin J, Zhou SZ, Wang J, Liu ZS, Liao J. Ketogenic diet group of pediatric neurology society of Chiese medical association. Therapeutic effects of ketogenic diet on human health [J]. Prospective multicenter study of long-term ketogenic diet for refractory epilepsy in children. Chin J Pediatr. 2013;51(4):276-82. https://doi.org/10.3760/cma.j.issn.0578-1310. 2013.04.008.

16. Li BM, Tong LL, Jia GJ, Lei GF, Yin P, et al. Short-term efficacy of ketogenic diet in the treatment of 31 children with refractory epileptic encephalopathy and its effect on electroencephalogram. Chin J Pediatr. 2013;51(005):362-6. https://doi.org/10.3760/cma.j.issn.0578-1310.2013. 05.009 .

17. Zhou YF, Gong XY, Zhou SZ, Wang Y, Chai YM, Yu LF,et al. Clinical characteristics and short-term efficacy of ketogenic diet in children with lateonset epileptic spasms. Chinese Journal of Evidence-based Pediatrics, 2015, 10(4):287-291.doi:3969/j.issn.1673-5501.2015.04.010.

18. Zhang J, Chen G, Wang J, Jiang Y, Yang Z, Xu K, et al. Efficacy of the ketogenic diet on ACTH- or corticosteroid-resistant infantile spasm: a multicentre prospective control study. Epileptic Disorders. 2021;23(2):337-45. https://doi.org/10.1684/epd.2021.1256.

19. Zhu XB, Chen O, Zhang DH, Jin RF, Li FH, Wang YB, et al. A prospective study on the treatment of infantile spasms with first-line topiramate followed by low-dose ACTH. Epilepsy Res. 2011;93(2-3):149-54. https://doi. org/10.1016/j.2010.12.002.

20. Sharma S, Sankhyan N, Gulati S, Agarwala A. Use of the modified Atkins diet in infantile spasms refractory to first-line treatment. Seizure. 2012;21(1):45-8. https://doi.org/10.1016/j.seizure.2011.08.009.
21. Caraballo RH, Cersosimo RO, Sakr D, Cresta A, Escobal N, Fejerman N. Ketogenic diet in patients with Dravet syndrome. Epilepsia. 2010;46(9):1539-44. https://doi.org/10.1111/j.1528-1167.2005.05705.x.

22. Kang HC, Yong JK, Dong WK, Kim HD. Efficacy and safety of the Ketogenic diet for intractable childhood epilepsy: Korean multicentric experience. Epilepsia. 2005;46. https://doi.org/10.1111/j.0013-9580.2005.48504.x.

23. Wu GF, Hu JS, Wu SH, Zhu HM, Mao B, Sun D, et al. Effect and retention rate of ketogenic diet on infantile spasms. Chin J Pract Pediatr. 2017;032(10):780-3. https://doi.org/10.3760/cma.j.issn.2095-428X.2017. 10.016 .

24. Zha J, Cao FF, Yi ZS, Li XY, Chen H, Sun XL, et al. Clinical observation of ketogenic diet in the treatment of 112 children with drug-resistant epilepsy. Jiangxi Med J. 2020;55(10):1499-502. https://doi.org/10.3969/j. issn.1006-2238.2020.10.050.
Ready to submit your research? Choose BMC and benefit from:

- fast, convenient online submission

- thorough peer review by experienced researchers in your field

- rapid publication on acceptance

- support for research data, including large and complex data types

- gold Open Access which fosters wider collaboration and increased citations

- maximum visibility for your research: over $100 \mathrm{M}$ website views per year

At BMC, research is always in progress.

Learn more biomedcentral.com/submissions 